\title{
La formación docente digital del profesor de Derecho en Cuba
}

\author{
Digital teacher training of the Law professor in \\ Cuba.
}

\section{Anaily Dayana Olazábal Revilla}

Profesora de Derecho de Autor y Derecho de Propiedad Industrial

Universidad de Camagüey, Cuba.

Maestrante en Ciencias de la Educación Superior y Doctoranda en Ciencias Pedagógicas

Email: anaily.olazabal@reduc.edu.cu

\begin{abstract}
Resumen: En la sociedad cubana actual, el Derecho y su enseñanza en las instituciones universitarias se ha visto fuertemente influenciado por fenómenos tales como la masificación del uso de la tecnología. Ante este acontecimiento, los docentes de Derecho deben poseer los conocimientos y habilidades necesarias para enfrentar con profesionalidad, las nuevas problemáticas que se suscitan tanto en la sociedad como en las universidades, escenarios donde las Tecnologías de la Información y la Comunicación (TIC) convergen como parte de la vida diaria de las personas. Se requiere, por tanto, la formación docente digital de los profesores de Derecho en pos de mejorar su desempeño docente. Motivo por el cual el presente tema se contextualiza en Cuba, en específico en la Universidad de Camagüey, donde los profesores del Departamento de Derecho presentan insuficiencias y limitaciones que obstaculizan su formación docente digital, las cuales son develadas por la autora.
\end{abstract}

Palabras clave: formación, formación docente digital, docente de Derecho, Universidad de Camagüey.

\begin{abstract}
In current Cuban society, Law and its teaching in university institutions has been strongly influenced by phenomena such as the mass use of technology. Faced with this event, law teachers must possess the knowledge and skills necessary to face professionally, the new problems that arise both in society and in universities, scenarios where Information and Communication Technologies (ICT) converge as part of the daily life of people. It is required, therefore, the digital teacher training of law professors in order to improve their teaching performance. Reason for which the present theme is contextualized in Cuba, specifically in the University of Camagüey, where the professors of the Law Department present insufficiencies and limitations that hinder their digital teaching training, which are unveiled by the author.
\end{abstract}

Keywords: training, digital teacher training, Law teacher, University of Camagüey. 


\section{La formación docente digital del profesor de Derecho en Cuba.}

Digital teacher training of the Law professor in Cuba.

\section{Introducción}

En la última década, la universidad cubana ha presenciado el impacto vertiginoso de un fenómeno tecnológico protagonizado por las Tecnologías de la Información y la Comunicación (TIC). Frente a este acontecimiento innovador, los docentes de Derecho enfrentan el reto de atemperarse a nuevas metodologías, concepciones renovadoras y nuevas tendencias que implican un cambio cualitativo en la formación del futuro jurista.

$\mathrm{Al}$ realizarse un breve recuento histórico de la enseñanza jurídica en Cuba, se destaca que la originaria Facultad de Leyes y Jurisprudencia fue una de las primeras que abrió sus puertas con la fundación de La Real y Pontificia Universidad de San Gerónimo de La Habana en 1728. Al respecto, Carranza (2016) afirma que desde su inicio y hasta la actualidad:

(...) la enseñanza y el aprendizaje del Derecho se ha caracterizado por ser una de las denominadas profesiones tradicionalista y que se ha visto fuertemente marcada por la cátedra magistral como un proceso pedagógico centrado en el docente, misma que se ha eternizado y no ha permitido un cambio a lo largo de los años. (p. 152).

Fruto de lo anterior, se llega a la conclusión de que la enseñanza de la Ciencia Jurídica en la casa de altos estudios, se caracteriza por la primacía de dos métodos fundamentales, dígase las conferencias magistrales y la solución de casos prácticos. Sin embargo, entre estos métodos tradicionales y las tendencias actuales de la Pedagogía se ha abierto una brecha protagonizada por la virtualidad.

De ello, es testigo perenne la carrera de Derecho en la Universidad de Camagüey, donde se implementa desde hace más de una década el curso a distancia. Modalidad de estudio que en la actualidad, se ha comenzado a fomentar con una fuerte tendencia hacia la virtualidad ${ }^{1}$. Por lo que los docentes, carentes de lo que Frosini (1983)

\footnotetext{
${ }^{1}$ Merchán, Mero, Mero y Antúnez (2017). Las infotecnologías y a la enseñanza virtual del Derecho. 112., Granma, Cuba.
} 
denominó conciencia informática ${ }^{2}$, precisan adaptarse a los nuevos escenarios de la educación jurídica propiciados por las TIC.

De manera que, desempeñar un rol activo en la nueva generación digital protagonizada por estudiantes nativos digitales, exige del docente de Derecho mucho más que un simple conocimiento básico en el uso del ordenador u otro dispositivo electrónico. Demanda comprender que:

La tarea de formar e informar a los juristas de estos tiempos debe ser una preocupación de todos, se trata de formar un profesional que esté vinculado a la problemática social, para que pueda comprender la naturaleza real del Derecho como ciencia, valorado como un fenómeno en sí mismo, donde se logre combinar propósitos prácticos y profesionales con otros estrictamente teóricos o científicos, como es el caso de la aplicación de las herramientas que nos aporta la informática. (Merchán, Mero, Mero y Antúnez, 2017, pág. 3).

Es necesario, por tanto, que los docentes reflexionen sobre la importancia que representan la tecnología y la Informática como nuevos escenarios que influyen positivamente en el campo del Derecho. Que participen "como conocedores activos y agentes críticos, usuarios proficientes de estos nuevos escenarios y posibilidades" (Abio, 2017, pág. 46).

Así, los profesores de Derecho de la universidad cubana, deben proyectar su formación docente digital hacia el logro de convertirse en profesores conocedores de la existencia y el manejo de los recursos tecnológicos e informáticos más avanzados. A fin de que puedan integrar el uso de dichos recursos como una herramienta más de su labor docente (Bocanegra, 2012).

Desde este punto de vista, el proceso de formación docente digital del profesor de Derecho ha sido estudiado por múltiples autores, entre los que puede citarse a Burgos (2009), Galindo (2010), Hurtado (2011), Rocha (2013), Ruiz (2013) y Gallego y Córdoba (2016). Por lo que se vislumbra la creciente necesidad de que el docente, asuma un rol protagónico en la integración de la tecnología a su labor.

\footnotetext{
${ }^{2}$ Consiste en la necesidad de actualizar los métodos y medios de enseñanza, de reconocer la existencia del vínculo indisoluble entre la Informática y el Derecho, así como saber emplear y explotar a cabalidad ese vínculo en la labor docente. Frosini, V. (1983). De la Informática Jurídica al Derecho Informático. Rivista Informatica e diritto, s.p. Obtenido de http://www.ittig.cnr.it/risorse/attivitaeditoriale/rivista/indici/
} 
No obstante, las investigaciones realizadas por los referidos autores, se han enfocado en la utilización de herramientas tecnológicas y digitales que permiten renovar la enseñanza jurídica. Por lo que asumen que los profesores, ya tienen una formación que les permite interactuar con las TIC en la esfera jurídica para su posterior traslado al escenario educativo. Cuando realmente, el proceso de formación en este ámbito es una de las principales necesidades y exigencias que enfrenta hoy el profesor de Derecho.

De tal suerte, los avances de informatización de la gestión judicial ${ }^{3}$ en la esfera internacional y nacional, los progresos referidos a la realización de audiencias virtuales, así como la participación activa en redes sociales y comunidades virtuales de temas jurídicos, son cuestiones muy puntuales que se escapan de las manos de los docentes. Consecuencia inmediata de su desconocimiento en el ámbito digital e informático, y su consiguiente interrelación con el Derecho.

Además, el acceso y tratamiento a bases de datos jurídicas, el uso de blogs jurídicos, el diseño de cursos de e-Derecho ${ }^{4}$, la realización de actividades colaborativas y aplicaciones didácticas para la enseñanza y difusión del Derecho, son acciones que no puede acometer el docente toda vez que carezca de saberes generales relacionados con el uso de la tecnología. Siendo las mismas, algunas de las principales afectaciones que impactan su labor y por consiguiente, la formación integral de los futuros egresados.

Los docentes del presente, deben ser conscientes de que la sociedad cubana no vive un cambio de era, sino más bien está inmersa en una era de cambios en la cual el jurista debe enfrentar relaciones civiles, penales, laborales, de propiedad industrial, etc., influenciadas por la innovación tecnológica (Rodríguez, Ricardo y Sariol, 2018). Así como asistir relaciones mercantiles, contractuales y comerciales en las cuales las TIC propician nuevos escenarios en el ámbito legal (Llaguno, 2012; Formentín, 2011 y 2013).

\footnotetext{
${ }^{3}$ Peche Becerra, M. D. (2012). Uso de las TIC en el Poder Judicial. Boletín Electrónico de la Organización de los Estados Americanos. OEA. Obtenido de http://www.ibader.info/wpcontent/uploads/Uso-de-las-TICs-en-el-Poder-Judicial.pdf

4 E-Derecho son tres modelos de cursos agrupados temáticamente: Asignatura, Seminario de Investigación y Comunicaciones, elaborados por profesores de Derecho, que permiten al profesor organizar y gestionar automatizadamente el soporte material y humano de la docencia, investigación y otras actividades universitarias. Flores, M. (2004). Enseñanza del Derecho con apoyo de las TIC. Proyecto interuniversitario e interdisciplinar. Revista Docencia y Capus Virtual, 63-66.
} 
Lo anterior permite develar que los profesores de Derecho de la universidad cubana presentan claras insuficiencias relacionadas con su desempeño docente ${ }^{5}$, dadas por el desconocimiento de la relación existente entre el Derecho y la Informática ${ }^{6}$, y la consecuente utilización de herramientas tecnológicas y digitales ${ }^{7}$ como un nuevo medio generador de conocimientos jurídicos.

En base a lo anterior, queda claramente demostrada la principal consecuencia de que los referidos sujetos carezcan de una formación docente digital, que les permita atemperar la enseñanza del Derecho a las necesidades actuales de formación del estudiantado.

Visto que estos últimos adolecerán de las habilidades y conocimientos necesarios para una vez en el ejercicio de la profesión, cumplir a cabalidad con su encargo social.

\section{Formación docente digital. Un análisis teórico necesario}

Según Benavides de Castañeda (2004) la tecnología y su consecuente uso en la esfera de la educación jurídica, ha servido como instrumento mediador tanto en la docencia como en la investigación. Dígase en la preparación de clases, en la creación de bases de datos, en el acceso a fuentes de información, así como en el uso de medios audiovisuales en el aula.

Por tanto, a criterio de la autora, la relación existente entre la enseñanza jurídica y los sujetos que intervienen en ella, requiere ser mediada por herramientas tecnológicas y digitales que faciliten una adecuada interacción y comunicación. Lo cual se corresponde en primera instancia, con los postulados planteados por Vygotsky (como se citó en Tejeda y Sánchez, 2008) al establecer que:

(...) el desarrollo del hombre está mediatizado por las relaciones interactivas que establece durante toda su vida, a través de las cuales se apropia y reproduce la cultura histórica de manera activa; es decir, todo lo que forma parte del mundo subjetivo, de lo intrapsíquico de cada sujeto, se expresará primero en la

\footnotetext{
${ }^{5}$ Tejedor y García (2010) consideran que el desempeño docente es cumplir con una responsabilidad en el trabajo docente. Donde se involucra de manera interrelacionada e intencionada los saberes, las habilidades, las actitudes y valores para el ejercicio de su función. Tejedor, J. y García, A. (2010). Evaluación del desempeño docente. Revista española de Pedagogía,(247), 439-459. Obtenido de https://dialnet.unirioja.es/servlet/articulo?codigo=3300545

${ }^{6}$ Fruto de esa relación nacen el Derecho Informático y la Informática Jurídica. El primero, dedicado a regular las leyes, normas y principios aplicables a los hechos y actos derivados de la informática. Mientras que la segunda, tiene como objeto la aplicación de la informática en el ámbito jurídico, dígase la recuperación y el tratamiento de información necesaria para tomar una decisión con consecuencias jurídicas.

${ }^{7}$ Dígase el uso de entornos personales de aprendizaje (PLE), el uso del campus virtual, el manejo de bases de datos jurídicas, el uso de objetos multimedia, utilización de la plataforma MOODLE, entre otros.
} 
intersubjetividad, para luego pasar de forma personalizada a cobrar sentido en el individuo; que tomará de la situación en función de sus necesidades, intereses, emociones y deseos. ( pág. 82).

Desde este punto de vista, el reconocimiento de esa relación mediada por la tecnología, provoca que surja la necesidad social de formar en los docentes de Derecho, "una actitud reflexiva, crítica y responsable ante los nuevos problemas que en las diversas esferas del acontecer social suscita la tecnología y ante los que ni el Derecho, ni quienes lo aplican o lo estudian pueden permanecer insensibles" (Pérez-Luño, 1996, p.60). Por tal motivo, la formación, como categoría indispensable de las Ciencias Pedagógicas, requiere un análisis previo que justifique su importancia en la presente investigación.

En un primer acercamiento a la formación, pudo constatarse que varios autores han ofrecido elementos sustanciales para su conceptualización (Zabalza, 2005; Tejeda y Sánchez, 2008; Fuentes, 2009; Rodríguez, Cabrera y Cruz, 2013). No obstante, la autora asume el concepto ofrecido por Águila, Ordan y Colunga (2017), al considerar que la misma:

Es un proceso dirigido a la preparación del ser humano como ser social de manera integral. Facilita que cada sujeto se apropie de manera diversa y única de parte de la cultura que le antecedió. Además, aprehende de su contexto a la vez que se instruye, educa y desarrolla. (p. 63).

Así pues, la formación desde su dimensión de proceso totalizador, ostenta un objetivo catalizador, el cual es preparar y formar al hombre en todos los aspectos de la vida. Dígase en el plano personal, emocional, así como en el ámbito social en lo referente al cumplimiento y respeto a determinadas conductas y normas sociales. Incluso a moldear en el individuo habilidades ${ }^{8}$, valores y conocimientos imprescindibles relacionadas con su profesión y el desempeño de la misma.

\footnotetext{
${ }^{8} \mathrm{La}$ autora asume que las habilidades son las estructuras psicológicas del pensamiento que permiten asimilar, conservar, utilizar y exponer los conocimientos. Se forman y desarrollan a través de la ejercitación de las acciones mentales y se convierten en modos de actuación que dan solución a tareas teóricas o prácticas. Álvarez (1996) citado por Mejías (2012), p. 19. Mejías Hernández, Y. (2012). Propuesta de actividades para desarrollar la habilidad intervención comunitaria en la asignatura Psicología Social II en estudiantes de cuarto año. Tesis de Maestría. Camagüey, Cuba: Universidad de Camagüey.
} 
En relación a lo anterior, se considera la formación docente como "ese proceso, formal o informal, de preparación profesional para el ejercicio de la praxis pedagógica y está ligado estrechamente a la experiencia en el aula" (Alves, 2003 citado por Zempoalteca, Barragán, González y Guzmán, 2017, p.84). No obstante, la formación docente a pesar de tener como base fundamental la experiencia del profesor en el aula, traspasa los límites del ámbito educativo.

Se consolida, por tanto, con las experiencias sociales y laborales adquiridas por el docente (Gallart, 1995). Ante lo cual, se coincide con Bajo (2005) quien afirma que "el capital humano no se forja solo en el sistema educativo" (p. 668) ya que reviste gran relevancia la influencia que la sociedad pueda ejercer en el docente, en vistas a lograr la preparación del mismo, para cumplir con el encargo social que le es encomendado por ésta.

No obstante, en la última década nuevos escenarios y espacios digitales han comenzado a invadir el ámbito académico. Ante este fenómeno tecnológico, los profesores precisan no solo de una formación propiamente docente, sino además, de una adecuada formación digital.

La autora asume el criterio de que el profesor de Derecho, no solo debe conocer y reconocer las potencialidades y ventajas que ofrecen los recursos digitales e informáticos a su propia labor. Sino que además, precisa emplear de forma didáctica esos recursos en el proceso docente educativo.

Por consiguiente, "se requiere formar docentes con los conocimientos y habilidades necesarios para la utilización de las TIC (...). Necesitan disponer, no solo de una alfabetización digital básica, sino también, ser capaces de integrar las TIC en sus prácticas didácticas" (Silva, Miranda, Gisbert, Morales, y Onetto, 2016, pág.57).

Desde este punto de vista, queda esclarecido que la formación docente debe incluir necesariamente dentro de su perfil, la integración de las TIC, consolidándose desde este ámbito, la formación docente digital (Matilla, Sayavedra y Alfonso, 2104; Viñals y Cuenca, 2016; Zempoalteca, Barragán, González y Guzmán, 2017). La cual, le permitirá al profesor universitario, resolver problemas asociados a:

(...) la investigación, la gestión, la innovación, la formación y evaluación, la responsabilidad ética y social, la construcción y difusión del conocimiento, así 
como la atención a los aspectos éticos, legales y ambientales que se deriven del uso de las TIC en la educación. (Rodríguez, 2014, p .6).

Por consiguiente, la autora asume la conceptualización ofrecida por Matilla, Sayavedra y Alfonso (2014) al considerar que la formación docente digital "implica conocer y usar diferentes herramientas TIC, reacomodar las prácticas; revisar y resignificar los conocimientos pedagógicos y disciplinares que le permitan al docente definir un ambiente de aprendizaje enriquecido con TIC" (p.12).

Lo anterior, se encuentra en total correspondencia con el Estándar de Formación

Docente en TIC, elaborado por la UNESCO en el año 2004. En base al cual, se advierte la necesidad e importancia de diseñar e implementar, estrategias y programas de capacitación docente, con el objetivo de que dichos sujetos logren utilizar las TIC de forma efectiva en el ámbito educativo.

\section{La formación docente digital de los profesores de Derecho en Cuba}

Una de las principales demandas y exigencias tanto de la institución universitaria como de la sociedad en este contexto, es la necesidad de que los docentes de Derecho en específico, sean formados en el ámbito de las TIC. Formación que les permitirá no solo, renovar la enseñanza del Derecho (Hurtado, 2011), sino que contribuirá al perfeccionamiento de la labor docente a la vez que se agilizará la inserción de los mismos en la informatización de la sociedad.

De tal suerte, varios autores han realizado pesquisas respecto a la formación docente digital de los profesores de Derecho. Entre los cuales, pueden citarse a Burgos (2009), Galindo (2010), Holgado (2011), Hurtado (2011), Ruiz (2013), Ávila (2015), Carranza (2016), Gallego y Córdoba (2016).

Las investigaciones y resultados aportados por los autores tributan en su mayoría, a la necesidad de que los profesores de Derecho implementen como parte de su labor docente el uso de entornos virtuales de aprendizaje. Así como también, la utilización de aplicaciones de la Web 2.0 en el proceso docente educativo.

De igual forma, los autores referenciados admiten la importancia de la formación del docente de Derecho en la sociedad del conocimiento9 ${ }^{9}$, así como la necesidad de

\footnotetext{
${ }^{9}$ Consiste en que las personas trabajen de manera colaborativa y realicen proyectos para tener niveles óptimos de calidad de vida en el marco de la inclusión, la convivencia pacífica, el desarrollo socioeconómico y la sustentabilidad ambiental, mediante la búsqueda, procesamiento, análisis,
} 
superación en el campo de las TIC. Reconociéndose de esta forma, que la superación en el ámbito pedagógico y disciplinar es insuficiente, toda vez que no se complemente con aquella destinada al uso de la tecnología.

Así pues, el primer jurista en reconocer la necesidad de que los docentes de Derecho se formen especialmente en el uso del ordenador, fue el italiano Frosini (1984, 1995,1996, 2000). Quien, desde una visión previsora, advierte que éstos necesitan reconocer el vínculo indisoluble que existe entre la Informática y las relaciones sociales y jurídicas.

No obstante, en el presente siglo no basta con que el docente de Derecho pueda desenvolverse con facilidad en el uso del ordenador. Sino que además, debe poseer un conjunto de destrezas que le permitan interactuar en las redes sociales y académicas, en los entornos virtuales de aprendizaje, buscar y seleccionar información en las bases de datos jurídicas, incluso que le faciliten la interacción y colaboración en la web $2.0^{10}$.

Desde este punto de vista, en la esfera internacional se han realizado múltiples investigaciones sobre este tópico, como la de Ruiz (2013) quien señala la importancia de incluir en la enseñanza del Derecho las herramientas de la Web 2.0. Para lo cual, reconoce en primera instancia, la necesidad de que el profesor reciba curos dirigidos a su formación docente digital.

Todo lo cual tributará a que el mismo, se convierta en un docente $2.0^{11}$. Logrando de esta manera “(...) no solo ser formado en materia de las diferentes áreas del Derecho, sino también en fortalecer las herramientas tecnológicas que le permitirán desempeñarse de manera efectiva en la actual sociedad moderna" (Carranza, 2016, p. 153).

Por consiguiente, la autora considera que la formación docente digital de los profesores de Derecho debe llevarse a cabo no solo por simples cuestiones metodológicas o estratégicas, sino además porque los mismos “deben formar parte del cambio, ser

adaptación, creación, innovación y aplicación del conocimiento, considerando diferentes fuentes y aplicando la tecnología. Tobón, S., Guzmán, C. E., Hernández, J. S. y Cardona, S. (2015). Sociedad del conocimiento: Estudio documental desde una perspectiva humanista y compleja. Paradigma, 36(2), 7-36. Obtenido de http://www.scielo.org.ve/scielo.php?script=sci

${ }^{10}$ La Web 2.0 es pensar, escribir, compartir y participar aprovechando al máximo las herramientas útiles que nos ofrece la Web. Los usuarios son los principales generadores de contenido. Usuarios y contenidos se pueden interconectar entre sí, de esta forma se crea una red. (Ruiz, 2013, p. 67)

11 Se describe el modelo de docente 2.0. como aquel que hace un uso efectivo de las herramientas que proporciona la Web 2.0 y con ello se convierte en un facilitador de contenidos, promueve el aprendizaje activo, es usuario de las tecnologías de la información y la comunicación (TIC), se atreve a ser aprendiz y colabora con los estudiantes. (Ruiz, 2013, p. 65) 
sujetos dinámicos, congruentes en su práctica y con la demanda de la sociedad" (Pérez, 2016, s.p).

No obstante, para cumplir con lo afirmado anteriormente, el docente de Derecho debe tener como premisa fundamental, adaptarse a los nuevos modelos de la educación influenciados por las TIC. Razón por la cual, se coincide con Lázaro, Gisbert y Silva (2018) al plantear que "el uso eficaz de las tecnologías digitales por parte del profesorado (...) implica movilizar conocimientos relacionados con las habilidades digitales básicas, con el uso didáctico de la tecnología y con la capacidad de seguir aprendiendo de manera continua." (p. 2).

De forma similar, De la Torre y Conde (2010) han realizado aportes encaminados a promover en el profesorado de Derecho, la formación docente asociada al manejo de las TIC. Enfatizando la necesidad de que éstos adquieran "destrezas tecnológicas relacionadas con la utilización de herramientas de computación y gestión de la información” (p. 4).

Por su parte, en el ámbito nacional uno de los antecedentes inmediatos de la formación de los docentes de Derecho en el ámbito de las TIC, lo constituye la celebración de la Sesión Científica de la Sociedad Cubana de Derecho e Informática. La cual, en el año 2015 dedica especialmente sus temas de debate a la necesidad de insertar a los profesionales del Derecho, especialmente a los docentes, en la informatización de la sociedad.

Desde este momento se impulsan en el territorio nacional la realización de eventos, conferencias magistrales, talleres, entre otras actividades destinadas a promover la formación de los docentes de Derecho en el ámbito de la innovación educativa y social. Con este propósito, se celebra en el año 2017 la XI Conferencia Internacional de Derecho e Informática de La Habana, en conjunto con el VII Congreso Iberoamericano de Investigadores y Docentes de Derecho e Informática.

Ambos eventos organizados por la UNESCO y la Unión Nacional de Juristas de Cuba, dan lugar al debate de temas tan novedosos como la enseñanza de la Ciencia Jurídica mediante la innovación didáctica. Fruto del cual, se comienza a cuestionar si el docente de Derecho, cuenta realmente con una formación pertinente para desempeñar un rol activo en la enseñanza virtual de esta ciencia (Antúnez, Antúnez y Soler, 2017; Merchán, Mero, Mero y Antúnez, 2017). 
En el mismo año 2017, la carrera de Derecho de la Universidad de Camagüey comienza a aunar esfuerzos para implementar el curso a distancia en la enseñanza de pregrado mediante el uso de la plataforma MOODLE. Lo cual constituye, uno de los primeros acercamientos a la enseñanza virtual del Derecho en la referida institución. Al respecto, Antúnez et al. (2017), plantean que:

La enseñanza virtual del Derecho, dentro del sistema pedagógico moderno, recurre a la tecnología informática para fortalecer el aprendizaje, la investigación, el autoestudio, la interacción y la flexibilidad de los estudios, permitiendo a los profesionales obtener una o varias profesiones, posgrados, especialidades, superando las barreras del tiempo y espacio para desarrollarse profesionalmente y contribuir al desarrollo de la sociedad. (p. 10).

No obstante, a pesar del gigantesco avance que representa para la carrera su implementación a través de una plataforma virtual, los docentes se muestran reticentes al cambio, a la vez que carecen de una adecuada preparación en el ámbito de las TIC para su aplicación a la enseñanza del Derecho.

Dígase, que los profesores de Derecho de la citada universidad, presentan ciertas insuficiencias y dificultades que les impiden llevar a cabo su formación docente digital, citándose entre ellas:

- Insuficientes saberes tecnológicos ${ }^{12}$ para proyectar un mejor desempeño docente en el ámbito de las TIC.

- Uso limitado de las TIC en el tratamiento didáctico de los contenidos, lo que manifiesta un arraigo a los medios tradicionales de la enseñanza del Derecho.

- Insuficiente preparación jurídico-informática ${ }^{13}$, lo que dificulta sus habilidades de utilizar la Informática como herramienta de asistencia al Derecho y su enseñanza.

\footnotetext{
12 Amador-Bautista (2003), considera que los saberes tecnológicos implican el saber recuperar, discriminar, seleccionar, interpretar, usar e intercambiar información; saber informarse, saber obtener información pertinente, saber hacer una buena utilización de la información disponible para comprender mejor el mundo en el que vivimos, así como comprender perfectamente los mecanismos de producción y de difusión de la información. Implica además la posibilidad de explotar creativamente la manipulación y el uso de las TIC desde el punto de vista didáctico, a través de la concepción, el diseño y la puesta en práctica de situaciones didácticas. pág. 107-109. Amador-Bautista, R. e. (2003). Tecnologías de información y comunicación. En Á. López y Mota, Saberes Científicos, Humanísticos y Tecnológicos: procesos de enseñanza y aprendizaje. Tomo II: Didáctica de las ciencias histórico-sociales. (págs. 1146). México: Consejo Mexicano de Investigación Educativa. Obtenido de http://www.acatlan.unam.mx

${ }^{13}$ Conjunto de conocimientos científicos y técnicas que hacen posible el tratamiento automático de la información jurídica por medio de ordenadores. Olivera, N. (2010). Estado de la cuestión en la relación entre Derecho e Informática. Revista Derecho y Nuevas Tecnologías, p. 508.
} 
- Falta de motivación e interés en cuanto a su actualización en las nuevas tendencias virtuales de la enseñanza de la Ciencia Jurídica ${ }^{14}$.

- Ausencia de acciones formativas dirigidas a potenciar el uso y aplicación de las TIC en el ámbito educativo.

A lo cual se suma la insuficiencia de recursos informáticos adecuados, lo que limita en gran medida la aplicación del nuevo modelo de educación a distancia basado en el uso de la plataforma MOODLE. Así como la interacción y comunicación entre docentes y estudiantes mediada por las TIC.

Por lo cual, urge que desde el propio Departamento de Derecho, se promueva la superación de los profesores en el ámbito digital. Que se convoquen cursos de posgrado, talleres, diplomados, entre otros, destinados a fomentar la formación docente digital del claustro.

Formación docente digital, que debe sustentarse en pilares que articulen los saberes pedagógicos generales, con los disciplinares y tecnológicos específicos. Teniendo como objetivo principal, la preparación del docente para que desarrolle un conjunto de acciones destinadas a incorporar las TIC como parte de su quehacer, y de las actividades desarrolladas en el aula.

Entre las acciones que pueden realizar los docentes de Derecho de la universidad cubana pueden citarse las siguientes:

- Desarrollar funciones de orientación, motivación o dinamización y de evaluación con el uso de las TIC (Delgado y Oliver, 2003).

- Usar medios tecnológicos y culturales más ágiles, que le permitan preparase para desenvolverse adecuadamente en los entornos virtuales (Benavides de Castañeda, 2004).

- Realizar la creación de la página web de la asignatura, la organización de la tutoría virtual con sus alumnos, el aprovechamiento de los recursos de Internet para las clases y para proponer actividades a los estudiantes (Marqués, 2008).

- Realizar proyectos colaborativos apoyándose en herramientas telemáticas, así como mantenerse al día a través de la documentación en red sobre las innovaciones tecnológicas, en la esfera educativa (Carrera y Coiduras, 2012).

\footnotetext{
${ }^{14}$ Requena, M. (2011). La docencia del Derecho a través de instrumentos interactivos:enseñar Derecho de la Unión Europea a través de la red. Revista Electrónica de Estudios Internacionales(11), 1-15.
} 
Lograr su superación en materia de inclusión de TIC en educación, para poder combinar sus conocimientos tecnológicos con su formación y experiencia en la docencia (Rocha, 2013).

- Asumir un nuevo rol del docente de la era 2.0 como organizador, guía, generador, acompañante, orientador, facilitador, tutor, dinamizador o asesor. Los cuales se asientan en la idea de cambiar la transmisión unidireccional del conocimiento por el intercambio horizontal de información (Viñals y Cuenca, 2016).

Con la implementación de las referidas acciones en la universidad camagüeyana, los docentes podrán instruirse en las nuevas tendencias de la informatización y la virtualidad en la enseñanza de la Ciencia Jurídica. Facilitándose, además, la comprensión de los diversos problemas jurídicos que se derivan de la influencia de la tecnología en el Derecho.

Por tanto, corresponde a la institución universitaria la ardua tarea de promover la formación de docentes de Derecho 2.0, capaces de conquistar los nuevos desafíos impuestos por las TIC. Visto que el reto que enfrentan los docentes de asumir el salto tecnológico es impresionante, y aquel que no sea capaz de sumarse estará dejando ir una de las más grandes oportunidades que ofrece el mundo moderno (Carbonell, 2011 citado por Pérez, 2016, p. 11).

\section{Conclusiones}

De la Torre y Conde (2010) consideran que la estrecha vinculación que existe entre el Derecho y las TIC es indiscutible, visto que el primero no queda ajeno al envite de la tecnología y a las profundas transformaciones que su incursión ha traído aparejada. Transformaciones que exigen del docente de Derecho, nuevos conocimientos y habilidades para su comprensión y enseñanza.

Desde este punto de vista, el estudio realizado sobre la formación docente digital de los docentes de Derecho en Cuba, ha permitido conocer en primera instancia, la importancia del referido proceso en la actualidad. Visto que el mismo contribuye significativamente no solo al mejoramiento del desempeño docente, sino además, a la renovación de la enseñanza del Derecho, así como a la comprensión de fenómenos jurídicos suscitados por la tecnología.

No obstante, la autora ha podido detectar que aunque los profesores de la universidad cubana poseen habilidades y conocimientos generales relacionados con las TIC, así 
como al uso del ordenador y otros dispositivos electrónicos, precisan de saberes en cuanto al manejo de herramientas digitales de la Web 2.0.

De igual forma, se requiere intencionar en el profesorado un cambio de mentalidad respecto al uso de las TIC en la docencia jurídica. Para lo cual deben concebirse desde el Departamento acciones que propicien mayor motivación y disposición de adquirir saberes relacionados con el uso didáctico de las TIC en el tratamiento de los contenidos.

Apremia, por tanto, trazar políticas institucionales que exijan la superación del profesorado en el campo de las TIC. Mediante lo cual se logrará la certera inserción de los docentes de Derecho, en la actual "sociedad y economía del conocimiento del siglo XXI” (Instituto Nacional de Tecnología Educativa y Formación del Profesorado [INTEF], 2017, pág. 5).

\section{Referencias bibliográficas}

Abio, G. (2017). Formación digital de profesores. Una revisión del tema con énfasis en los modelos de competencia/literacidades digitales. Caracol(13), 21-55. Obtenido de: https://www.researchgate.net/publication/315729728_Formacion_digital_de_pro fesores_Una_revision_del_tema_con_enfasis_en_los_modelos_de_competencia sliteracidades_digitales?enrichId=rgreq-c4e2ccfcde0c4b911c1ac61af0eca7e6$\underline{X X X \& \text { enrichSource }=\text { Y292ZXJQYWdlOzMxNTc }}$

Águila, A., Ordan, A. I. y Colunga, S. (2017). Acercamiento teórico a la formación de las competencias emocionales en los alumnos de la carrera arte danzario, perfil ballet. Didáctica y Educación., VIII(1), 61-74. Obtenido de https://www.researchgate.net/publication/321368910_ACERCAMIENTO_TEO RICO_A_LA_FORMACION_DE_LAS_COMPETENCIAS_EMOCIONALES EN_LOS_ALUMNOS_DE_LA_CARRERA_DE_ARTE_DANZARIO_PERF IL_BALLET?enrichId=rgreq-10eac335de1 cacbfd2e9368cd57a869b$\underline{\text { XXX\&enrichSource }=\text { Y292ZXJQYWd }}$

Antúnez, A., Antúnez, A. y Soler, Y. (2017). La enseñanza virtual del Derecho. Una aproximación en la universidad cubana. Revista de Educación y Derecho(16), 222. Obtenido de http://urbeetius.org/wp-content/uploads/2017/05/LaEnse\%C3\%B1anza-Virtual-del-Derecho-en-la-Universidad-Cubana.pdf 
Ávila, M. G. (2016). Enseñar a aprender en Derecho: la importancia de la formación docente del formador. 1-13. Obtenido de http://www.google.com/url?q=https://dialnet.unirioja.es/descarga/articulo/48462 81.pdf\&sa=U\&ved=2ahUKEwiAz7z87J_gAhUpqlkKHWWKBU8QFjAAegQI BhAB\&usg=AOvVaw3AggWSu2AzfAqMGcDOJzY7

Bajo, N. (2005). Educación, economía global y mercado global. Anuario Jurídico y Económico Escurialense, XXXVIII, 641-670. Obtenido de http://www.google.com/url?q=https://dialnet.unirioja.es/descarga/articulo/11430 78.pdf\&sa=U\&ved=2ahUKEwiIhL_N7Z_gAhWwq1kKHXi5BFcQFjAAegQIB $\underline{\text { RAB\&usg=AOvVaw13Q3_OXXt_ZSy5VmQ0WNxV }}$

Benavides de Castañeda, L. E. (2004). Reduciendo la brecha digital: enseñanza del Derecho en entorno Web. Ponencia en Congreso EDUTEC 2004. Barcelona, España. Obtenido de http://www.lmi.ub.es/edutec2004/pdf/161.pdf

Bocanegra, H. (2012). La enseñanza del Derecho y la formación de los abogados. Revista Republicana(12), 323-347. Obtenido de http://www.google.com/url?q=http://revista.urepublicana.edu.co/wpcontent/uploads/2012/07/La-ense\%25C3\%25B1anza-del-derecho-y-laformacion-de-losabogados.pdf\&sa=U\&ved=2ahUKEwiW9MWG7p_gAhWKjFkKHZsNB04QFj $\underline{\text { AAegQICBAB\&usg=AOvVaw1DjyDkXBcJqyHiEWfPN1Jj }}$

Burgos, M. (2009). La formación del jurista en la sociedad del conocimiento. Un análisis desde la dialéctica. Reshes. Revista de Derechos Humanos y Estudios Sociales(1), $127-148$. Obtenido de http://www.google.com/url?q=http://www.academia.edu/7635360/La_formaci\% 25C3\%25B3n_del_jurista_en_la_sociedad_del_conocimiento._Un_an \%25C3\%2 5A1lisis_desde_la_cr\%25C3\%25ADtica_dial\%25C3\%25A9ctica_2009\&sa=U\& ved=2ahUKEwjy3py97p_gAhVrw1kKHbhGCUcQFjAAegQIARAB\&usg=AOv Vaw2k_lwJK1yCRUj4ivh7UKwO

Carranza, M. d. (2016). El uso de las TIC en la enseñanza y el aprendizaje del Derecho. Revista educ@rnos, 151-168. Obtenido de https://www.researchgate.net/profile/Maria_Alcantar/publication/317571019_El _uso_de_las_TIC_en_la_ensenanza_y_el_aprendizaje_del_Derecho/links/59405 
e02aca272371224e3f3/El-uso-de-las-TIC-en-la-ensenanza-y-el-aprendizaje-delDerecho.pdf?origin=publication_d

Carrera, J. y Coiduras, J. (2012). Identificación de la competencia digital del profesorado universitario: un estudio exploratorio en el ámbito de las Ciencias Sociales. Revista de Docencia Universitaria, 10, 273-298. Obtenido de $\underline{\text { www.redaberta.usc.es }}$

De la Torre, F. y Conde, P. (2010). Propuestas para la aplicación de las nuevas tecnologías a las enseñanzas jurídicas de grado y postgrado desde la perspectiva de Bolonia. Obtenido de http://www.uoc.edu/symposia/dretitic/pdf/5_fco_de_la_torre.pdf

Delgado, A. M. y Oliver, R. (2003). Enseñanza del Derecho y tecnologías de la información y la comunicación. Cataluña, España. Obtenido de http://www.uoc.edu/dt/20310/index.html

Formentín, Y. (2011). La sociedad de la información y la contratación vía electrónica: Retos del Siglo XXI. Contribuciones a las Ciencias Sociales. Obtenido de http://www.google.com/url?q=http://www.eumed.net/rev/cccss/14/ymfz.html\&s

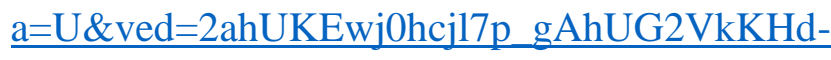

\section{$\underline{\text { RCFkQFjAAegQIBxAB\&usg=AOvVaw02FnUh8ZQvHoCXIriOc6QY }}$}

Formentín, Y. (2013). La firma electrónica, su recepción legal. Especial referencia a la ausencia legislativa en Cuba. Revista del instituto de Ciencias Jurídicas de Puebla. (31), pp 104-120. Obtenido de http://www.google.com/url?q=https://dialnet.unirioja.es/descarga/articulo/46461 85.pdf\&sa=U\&ved=2ahUKEwjd64eL75 gAhVB1VkKHV9kDE0QFjAAegQIA $\underline{\text { RAB\&usg=AOvVaw080B7u_4ZQyLY6cRX5iGG9 }}$

Frosini, V. (1983). De la Informática Jurídica al Derecho Informático. Rivista Informatica e diritto, s.p. Obtenido de http://www.ittig.cnr.it/risorse/attivitaeditoriale/rivista/indici/

Frosini, V. (1984). Le droit de l'informatique dans les années 80. Rivista "Informatica e diritto", $\quad X(2), \quad 63-72 . \quad$ Obtenido de www.ittig.cnr.it/risorse/attivitaeditoriale/rivista/indici/2-1984/ 
Frosini, V. (1995). Riflessioni sui contratti d'informatica. Rivista Informatica e diritto, s.p. Obtenido de http://www.ittig.cnr.it/risorse/attivita-editoriale/rivista/indici/

Frosini, V. (1996). Towards Information Law. Rivista Informatica e diritto, s.p. Obtenido de http://www.ittig.cnr.it/risorse/attivita-editoriale/rivista/indici/

Frosini, V. (2000). El jurista en la sociedad tecnológica. Argumentos de Razón Técnica(2), 121-136. Obtenido de http://www.google.com/url?q=https://idus.us.es/xmlui/handle/11441/21687\&sa=U\&v ed=2ahUKEwjTgvo75 gAhUEvlkKHYIDDFMQFjABegQICRAB\&usg=AOvVaw3IVdvMwFtyL6orDS8IW2gS

Fuentes, H. (2009). Formación por competencias. Venezuela: Editorial Guaranda. Universidad Estatal de Bolivar.

Galindo, F. (2010). La formación del jurista y las TIC. 317-331. Zaragoza, España. Obtenido de http://www.juridicas.unam.mx/

Gallart, M. A. (1995). Cuestiones actuales de la formación. (R. Bertoncello, Ed.) $\begin{array}{lllll}\text { Boletín } & \text { y } & \text { Trabajo. } & \text { Obtenido }\end{array}$ https://www.researchgate.net/publication/44822165_Cuestiones_actuales_de_la formacion

Gallego, J. E. y Córdoba, M. M. (2017). Una aproximación a la incorporación de TIC en la formación de abogados. Clínica Jurídica de Interés Público UNAULA, 3(5), 27-56. Obtenido de http://www.google.com/url?q=http://scienti.colciencias.gov.co:8081/cvlac/visual izador/generarCurriculoCv.do\%3Fcod_rh\%3D0000701530\&sa=U\&ved=2ahUK Ewj5tuqE8J_gAhUC2VkKHQnEDUwQFjALegQIBRAB\&usg=AOvVaw0DGk TrbCtbgxPY5FQMvq0M

Holgado, C. (2011). Las nuevas tecnologías en los estudios de Derecho en el marco del EESS: sugerencias didácticas de actividades colaborativas con entornos virtuales. REJIE: Revista Jurídica de Investigación e Innovación Educativa(3), 93-106. Obtenido de http://www.eumed.net/rev/rejie

Horruitiner, P. (2011). La educación superior. Retos y perspectivas en la educación superior cubana. La Habana, Cuba: MES. Obtenido de http://www.google.com/url?q=http://beduniv.reduniv.edu.cu/fetch.php\%3Fdata 
\%3D274\%26type\%3Dpdf\%26id\%3D2887\%26db\%3D0\&sa=U\&ved=2ahUKEw inyvy_8J_gAhXIzlkKHSmhAUsQFjAEegQIBBAB\&usg=AOvVaw3SkV0gzju3izRgoJbQn6G

Hurtado, L. F. (2011). Renovación de las prácticas pedagógicas con la inserción de las TIC: aplicación del modelo b-learning en la enseñaza del derecho. Memorando de Derecho, 13(21), 49-62. Obtenido de https://dialnet.unirioja.es/descarga/articulo/3730975.pdf

INTEF. (2017). Marco Común de Competencia Digital Docente. España: Instituto nacional de Tecnología Educatva y Formación del Profesorado. Obtenido de http://aprende.educalab.es

Lázaro, J. L., Gisbert, M., \& Silva, J. E. (2018). Una rúbrica para evaluar la competencia digital del profesor universitario en el contexto latinoamericano. Revista Electrónica de Tecnología Educativa.(63). Obtenido de https://www.researchgate.net/publication/324073863_Una_rubrica_para_evaluar _la_competencia_digital_del_profesor_universitario_en_el_contexto_latinoamer icano?enrichId=rgreq-0e7e0a23cb17eb1ac40bea4ce40f96cb$\underline{\text { XXX\&enrichSource }=Y 292 Z X J Q Y W d 1 O z M y N D A 3 M z g 2 M z t B U z o 2 M}$

Llaguno, D. (2012). La fiscalidad del comercio electrónico en el entorno tributario cubano. Revista DIXI. Diciembre 2012. At. 86. Obtenido de http://www.google.com/url?q=https://revistas.ucc.edu.co/index.php/di/article/vie w/32/33\&sa=U\&ved=2ahUKEwiMzZPt8J_gAhVkvFkKHYRyCUcQFjAAegQI CBAB\&usg=AOvVaw1_J5NOu94y9oarp7-FndF

Marqués, P. (17 de abril de 2008). Las competencias digitales de los docentes. Recuperado el 12 de febrero de 2017, de Universidad Autónoma de Barcelona: http://www.slideshare.net/peremarques/claves-para-la-integracin-educativa-de$\underline{\text { las-tic }}$

Matilla, M., Sayavedra, C. y Alfonso, V. C. (2014). Competencias TIC en alumnos universitarios: Dimensiones y categorías para su análisis. Trabajo presentado en el Congreso Iberoamericano de Ciencia, Tecnología, Innovación y Educación. 1-16. Buenos Aires, Argentina. Obtenido de http://www.google.com/url?q=https://www.oei.es/historico/congreso2014/memo riactei/1534.pdf\&sa=U\&ved=2ahUKEwiE-K- 
$\underline{\text { n8Z_gAhXGq1kKHVh8CS0QFjAAegQIBxAB\&usg=AOvVaw2MLlm9xw82ft }}$ $\underline{\text { hglnUefM3D }}$

Mejías, Y. (2012). Propuesta de actividades para desarrollar la habilidad intervención comunitaria en la asignatura Psicología Social II en estudiantes de cuarto año. Tesis de Maestría. Camagüey, Cuba: Universidad de Camagüey.

Merchán, E., Mero, K., Mero, C. y Antúnez, A. (2017). Las infotecnologías y a la enseñanza virtual del Derecho. 1-12., Granma, Cuba. Obtenido de http://www.pensamientopenal.com.ar/doctrina/45405-infotecnologias-yensenanza-virtual-del-derecho

Ojeda, Z. y Amoroso, Y. (2016). La protección de los datos personales en Cuba desde la legislación vigente. Justicia Juris, 12 (2),87- 94. Obtenido de http://www.google.com/url?q=https://www.researchgate.net/publication/313482 122_La_proteccion_de_los_datos_personales_en_Cuba_desde_la_legislacion_vi gente1\&sa=U\&ved=2ahUKEwju7PTI8Z_gAhWH11kKHbiiDFAQFjABegQIBx AB\&usg=AOvVaw1IKwhsUX5AeMyv4nCYu_Bk

Pérez, S. V. (1ro de noviembre de 2016). Abogados y estudiantes de Derecho frente a las TIC. Recuperado el 26 de noviembre de 2017, de AZ Portal de Educación y Cultura de México: http://www.educacionyculturaaz.com/

Pérez-Luño, A. (1996). Manual de informática y derecho. Barcelona: Editorial Ariel S.A. Obtenido de https://rua.ua.es/dspace/bitstream/10045/10359/1/doxa212_15.pdf

Rocha, D. (2013). Formación docente e innovación pedagógica: el desafío de la incorporación de las Tic's a la enseñanza del derecho. Ponencia. Buenos Aires, Buenos Aires, Argentina: Universidad de Buenos Aires. Obtenido de http://www.google.com/url?q=https://www.researchgate.net/publication/282556 250_Pedagogia_Efectiva_e_Innovaciones_en_la_Formacion_de_Periodistas_Int egrados\&sa=U\&ved=2ahUKEwiEjtjq8Z_gAhWnrFkKHU2iB1sQFjABegQICB AB\&usg=AOvVaw1zTjYPnFrkGFy9QNH9bGAd

Rodríguez, I. (2014). Propuesta de un modelo para un programa de capacitación docente en competencias digitales. Revista Iberoamericana de Producción Académica y Gestión Educativa, 1-13. Obtenido de http://www.google.com/url?q=https://www.pag.org.mx/index.php/PAG/article/v 


\section{iewFile/54/97\&sa=U\&ved=2ahUKEwj4i7ig8p_gAhWntlkKHUQ-}

\section{C0oQFjAAegQIARAB\&usg=AOvVaw1kzo6XRgTjM9HS_gD-LQ_W}

Rodríguez, M., Cabrera, E. y Cruz, J. (2013). La utilización de la categoría formación en la investigación en Ciencias Pedagógicas. 1-12. Obtenido de http://www.google.com/url?q=http://atlante.eumed.net/cienciaspedagogicas/\&sa=U\&ved=2ahUKEwjHmpnJ8p_gAhULtlkKHQDaCk0QFjAAe gQICRAB\&usg=AOvVaw2UGLbSASjm_C4uE36T67Rm

Rodríguez, Y., Ricardo, R. y Sariol, Y. (2018). Sistema de gobierno electrónico en la República de Cuba. Trabajo presentado en el V Taller Internacional las TIC en la Gestión de las Organizaciones. La Habana, Cuba. Obtenido de https://dialnet.unirioja.es/descarga/articulo/3730975.pdf

Ruiz, N. (2013). Docente 2.0- Inclusión de TIC's en el aula. Revista Carrera y Formación Docente(2), 65-72. Obtenido de www.derecho.uba.ar/revista-carreradocente

Silva, J., Miranda, P., Gisbert, M., Morales, J., \& Onetto, A. (2016). Evaluación de la competencia digital en la formación inicial docente: Una propuesta en el contexto Chileno - Uruguayo. Revista Latinoamericana de Tecnología Educativa, $\quad$ 15, 55-68. Obtenido de http://www.google.com/url?q=https://www.researchgate.net/publication/273440 992_Competencia_digital_docente_desempenos_didacticos_en_la_formacion_in cial_del_profesorado\&sa=U\&ved=2ahUKEwj6j9_s8p_gAhWpxVkKHe6mCUw QFjAAegQIBxAB\&usg=AOvVaw2WZYiszBBq7v-LUv_cyARF

Tejeda, R. y Sánchez, P. (2008). La formación basada en competencias en los contextos universitarios. Holguín, Cuba: Universidad de Holguín.

UNESCO. (2004). Las tecnologías de la información y la comunicación en la formación docente. Montevideo, Uruguay: UNESCO.

Viñals, A. y Cuanca Amigo, J. (2016). El rol del docente en la era digital. Revista Interuniversitaria de Formación del Profesorado, 30(2), 103-114. Obtenido de http://www.redalyc.org/articulo.oa?id=27447325008

Vygotsky, L. S. (1985). Historia del desarrollo de las funciones psíquicas superiores. La Habana: Editorial Científico-Técnica. Obtenido de 

203.pdf?

Zabalza, M. Á. (2005). Competencias docentes. Competencias docentes (págs. 87-125). Santiago de Compostela: Universidad de Santiago de Compostela. Obtenido de http://saladeaulainterativa.pro.br/moodle/mod/resource/view.php?inpopup=true $\underline{\text { \&id }=3711}$

Zempoalteca, B., Barragán, J. F., González, J., y Guzmán, T. (2017). Formación en TIC y competencia digital en la docencia en instituciones públicas de educación superior. Revista Apertura, 9(1), 80-96. Obtenido de http://dx.doi.org/10.18381/Ap.v9n1.922 